\title{
Coupling of Caged Molecule Dynamics to JG $\beta$-Relaxation: I
}

\author{
S. Capacciolia ${ }^{\mathrm{a}, \mathrm{b}^{*}}$, K.L. Ngai ${ }^{\mathrm{b}, \mathrm{c}^{*}}$, M. Shahin Thayyil ${ }^{\mathrm{d}}$, D. Prevosto ${ }^{\mathrm{b}}$ \\ ${ }^{a}$ Dipartimento di Fisica, Università di Pisa, Largo Bruno Pontecorvo 3 ,I-56127, Pisa, Italy \\ ${ }^{\mathrm{b}}$ CNR-IPCF, Largo Bruno Pontecorvo 3 ,I-56127, Pisa, Italy \\ ${ }^{c}$ State Key Lab of Metastable Materials Science and Technology, \\ Yanshan University, Qinhuangdao, Hebei, 066004 China, \\ ${ }^{\mathrm{d}}$ Department of Physics, University of Calicut, Kerala, INDIA \\ *corresponding authors: simone.capaccioli@df.unipi.it, Kia.Ngai@pi.ipcf.cnr.it
}

\begin{abstract}
The paper [Juraj Sibik, Stephen R. Elliott, and J. Axel Zeitler, J. Phys. Chem. Lett. 2014, 5, 1968-1972] used terahertz time-domain spectroscopy (THz-TDS) to study the dynamics of the polyalcohols, glycerol, threitol, xylitol, and sorbitol, at temperatures from below to above the glass transition temperature $T_{g}$. On heating the glasses, they observed the dielectric losses, $\varepsilon^{\prime \prime}(v)$ at $v=1 \mathrm{THz}$, increase monotonically with temperature and change dependence at two temperatures, first deep in the glassy state at $T_{T H z}=0.65 T_{g}$, and second at $T_{g}$. The effects at both temperatures are most prominent in sorbitol but become progressively weaker in the order of xylitol and threitol, and the sub- $T_{g}$ change was not observed in glycerol. They suggested this feature originates from the high-frequency tail of the JohariGoldstein (JG) $\beta$-relaxation, and the temperature region near $0.65 T_{g}$ is the universal region for the secondary glass transition due to the JG- $\beta$ relaxation. In this paper we first use isothermal dielectric relaxation data at frequencies below $10^{6} \mathrm{~Hz}$ to locate the 'second glass transition' temperature $T_{\beta}$ at which the JG $\beta$-relaxation time $\tau_{J G}$ reaches $100 \mathrm{~s}$. The value of $T_{\beta}$ is close to
\end{abstract}


$T_{T H z}=0.65 T_{g}$ for sorbitol $\left(0.63 T_{g}\right)$ and xylitol $\left(0.65 T_{g}\right)$, but $T_{\beta}$ is $0.74 T_{g}$ for threitol and $0.83 T_{g}$ for glycerol. Notwithstanding, the larger values of $T_{\beta}$ of glycerol is consistent with the THzTDS data. Next, we identify the dynamic process probed by THz-TDS as the caged molecule dynamics, showing up in susceptibility spectra as nearly constant loss (NCL). The caged molecule dynamics regime is terminated by the onset of the primitive relaxation of the Coupling Model, which is the precursor of the JG $\beta$-relaxation. From this relation, established is the connection of the magnitude and temperature dependence of the NCL and those of $\tau_{J G}$. This connection explains the monotonic increase of NCL with temperature and change to a stronger dependence after crossing $T_{\beta}$ giving rise to the sub- $T_{g}$ behavior of $\varepsilon^{\prime \prime}(v)$ observed in experiment. Beyond the polyalcohols, we present new dielectric relaxation measurements of flufenamic acid and recall dielectric, NMR and calorimetric data of indomethacin. The data of these two pharmaceuticals enables us to determine the value of $T_{\beta}=0.67 T_{\mathrm{g}}$ for flufenamic acid and $T_{\beta}=0.58 T_{\mathrm{g}}$ or $T_{\beta}=0.62 T_{\mathrm{g}}$ for indomethacin, which can be compared with experimental values of $T_{T H z}$ from THz-TDS measurements when become available. We point out that the sub- $T_{g}$ change of NCL at $T_{\beta}$ found by THz-TDS can be observed by other high frequency spectroscopy including neutron scattering, light scattering, Brillouin scattering, and inelastic X-ray scattering. An example from neutron scattering is cited. All the findings demonstrate the connection of all processes in the evolution of dynamics ending at the structural $\alpha$ relaxation.

Keywords: THz absorption, dielectric spectroscopy, Johari-Goldstein , fast dynamics, nearly constant loss, polyalcohols 


\section{Introduction}

The high frequency measurements of in the gigahertz to terahertz range have been a major area of activity in research on the dynamics in glass-forming systems to join in the effort to solve the glass transition problem. Techniques used including quasielastic neutron scattering ${ }^{1-}$

${ }^{11}$, Brillouin light scattering ${ }^{12}$, dynamic light scattering ${ }^{13-17}$, inelastic X-ray scattering ${ }^{18-20}$, extraordinary high frequency dielectric spectroscopy up to a few hundred gigahertz ${ }^{21-23}$, and terahertz time-domain spectroscopy ${ }^{24-27}$. The counterparts of the high frequency measurements are the time domain optical heterodyne detected optical Kerr effect experiments from $1 \mathrm{ps}$ to tens of $\mathrm{ns}^{28}$, and molecular dynamics simulations ${ }^{30-33}$. There are some studies attempting to link dynamic quantities measured at higher frequencies/short times to variables either determining the glass transition or quantifying some salient properties at the glass transition temperature $T_{g}$. One example is the mean-square-displacement (MSD), $\left\langle u^{2}(T)>\right.$, measured by quasielastic neutron scattering (QENS) at temperatures below and above $T_{g}$. Found generally in glass-formers of different kinds, smaller molecular, polymeric, and inorganic alike, is the change of the temperature dependence of $\left\langle u^{2}(T)\right\rangle$ at $T_{g}$. The remarkably weak $T$-dependence of $\left\langle u^{2}(T)\right\rangle$ at temperatures below $T_{g}$ changes to a stronger $T$ dependence above $T_{g}{ }^{2,3,6-11,34,35,36}$ Moreover $^{34}$, measured by spectrometer with the same resolution, $\left\langle u^{2}(T)\right\rangle$ is larger and the change at $T_{g}$ is sharper if the structural $\alpha$-relaxation of the glass-former has broader frequency dispersion or smaller value of the exponent, $(1-n)$, of its Kohlrausch correlation function,

$$
\phi(t)=\exp \left[-\left(t / \tau_{\alpha}\right)^{1-n}\right]
$$


where $\tau_{\alpha}$ is the $\alpha$-relaxation time. Another notable example is the inelastic x-ray scattering (IXS) measurements of the dynamic structure factor and the determination of the nonergodicity factor $f(Q, T)$ by Scopigno and coworkers ${ }^{19}$. They found in glass-formers a correlation between the temperature dependence of $f(Q, T)$ in the glassy state and fragility ${ }^{35,36}$. The latter is the rate of increase of $\log _{10} \tau_{\alpha}$ as function of the $T_{g} / T$ on cooling at $T_{g} / T=1$. This correlation from experiment was confirmed by molecular dynamics simulations of the binary Lennard-Jones particles systems with different interaction potentials ${ }^{30}$.

These two examples, i.e. the $T$-dependences of $\left\langle u^{2}(T)\right\rangle$ and $f(Q, T)$, together with others not mentioned are empirical facts suggesting that the fast processes somehow are related to the slow dynamics. This connection was made explicit in the Coupling Model $(\mathrm{CM})^{37-39}$ specifically in considering the dynamics evolving with time in the order of the caged molecules, the primitive relaxation which is a part of the Johari-Goldstein (JG) $\beta$-relaxation, and the structural $\alpha$-relaxation ${ }^{37-44}$. Initially at short times molecules are caged. In this caged dynamics regime, the susceptibility, $\chi^{\prime \prime}(T, v)=B(T)(v)^{-\lambda}$, originates from the dissipation of movements confined by the anharmonic potential. Its weak temperature dependence $B(T)$ is well described by $A \exp \left(T / T_{0}\right)$. Its power law frequency dependence with $\lambda<<1$ leads to the term of the nearly constant loss (NCL). The NCL is not a relaxation process with a characteristic time. It persists to lower frequencies/longer times until the atoms, or molecules leaves the cage. It is the first or primitive relaxation with relaxation time, $\tau_{0}$, that effectively terminates the caged dynamics regime, and the NCL dependence of the susceptibility no longer holds at frequencies of the order of $v_{0}=1 /\left(2 \pi \tau_{0}\right){ }^{37,39,45,46}$ Of particular interest is ref.46 where the experimental results presented therein are for the polyalcohols. Ions in molten and glassy ionic conductors caged at short times exhibit the NCL, which is also terminated by the 
primitive ion hop frequency $v_{0}{ }^{47-50}$ The paper ${ }^{51}$ shows the remarkable similarities, and also a correlation of the magnitude of the NCL with the primitive relaxation time $\tau_{0}$ or approximately the JG $\beta$-relaxation time $\tau_{\mathrm{JG}}$ to be defined later

This relation between the NCL and the primitive relaxation has the consequence of any change of the primitive relaxation is reflected by the corresponding change of the NCL, and vice versa. The sequential relation of the primitive relaxation or the JG $\beta$-relaxation with the $\alpha$-relaxation is the core result of the $\mathrm{CM}$, which has been confirmed in various ways and in many glass-forming systems ${ }^{52-57}$, and in the analogue ionic systems ${ }^{58-59}$.

A recent paper by Sibik et al. ${ }^{60}$ used terahertz time-domain spectroscopy (THz-TDS) in the frequency range of $0.2-3 \mathrm{THz}$ to study the dynamics of the family of polyalcohols, glycerol, threitol, xylitol, and sorbitol, at temperatures between 80 and $310 \mathrm{~K}$. On heating the glasses, they observed the dielectric losses, $\varepsilon^{\prime \prime}(v)$ at $v=1 \mathrm{THz}$, increase monotonically with temperature but the temperature dependence changes at two temperatures, first deep in the glassy state at a sub- $T_{g}$ temperature of $0.65 T_{g}$, and second at $T_{g}$. The effects at both temperatures are most prominent in sorbitol but become progressively weaker in the order of xylitol and threitol, and the sub- $T_{g}$ change was not observed in glycerol. They suggested this feature originates from the high-frequency tail of the JG $\beta$ relaxation, and the temperature region near $0.65 T_{g}$ is the universal region for the secondary glass transition due to the JG- $\beta$ relaxation. Also they proposed that the thermal changes in the losses near $0.65 T_{g}$ seem to be underpinned by a universal change in the hydrogen bonding structure of the samples. The study by THz-TDS has been extended to several pharmaceutical glass-formers including flufenamic acid and indomethacin. ${ }^{61}$ 
In this paper, we critically reexamine the experimental data of the polyalcohols by Sibik et al. and relate the observed $\varepsilon^{\prime \prime}(v)$ at $v=1 \mathrm{THz}$ to the proper dynamic process responsible for its changes of temperature dependences at sub- $T_{g}$ temperature and at $T_{g}$. By analyses of the dielectric relaxation spectra of the polyalcohols, we identify the proper dynamic process is the NCL of caged molecules. We provide dielectric measurements of flufenamic acid and indomethacin to characterize the $\alpha$-relaxation and the JG $\beta$-relaxation, and determine their relaxation time as well as that of primitive relaxation time. The results are used to obtain the value of $T_{\beta}$ to predict the temperature of the sub- $T_{g}$ change of temperature dependence of $\varepsilon^{\prime \prime}(v)$ at $v=1 \mathrm{THz}$ in THz-TDS experiment. Previously published data of indomethacin are employed to make the same prediction for this pharmaceutical. In the final part of the paper, theoretical arguments supported by experimental facts are given to demonstrate the inseparable link of the NCL of caged molecules to the primitive relaxation and the JG $\beta$ relaxation. There is a change of the temperature dependence of the JG $\beta$-relaxation at $T_{\beta}$ below which it falls out of equilibrium. Due to the linkage, the change is transferred to the corresponding change of the temperature dependence of the NCL. Justification of this explanation by theory with support from other experimental facts is given.

\section{Experimental Details}

Sorbitol and flufenamic acid were purchased in anhydrous form at high purity grade from Sigma-Aldrich and used as received, with the precaution of storing and handling them under dry atmosphere. A parallel plate capacitor separated by a quartz spacer and filled by the sample was placed in the nitrogen flow Quatro cryostat. Glassy and supercooled systems were obtained by heating few degrees above the melting point under nitrogen atmosphere and then cooling. Novocontrol Alpha-Analyzer was used for dielectric measurements from $1 \mathrm{mHz}$ to 
$10 \mathrm{MHz}$, whereas for higher frequencies $(1 \mathrm{MHz}-6 \mathrm{GHz})$ measurements have been carried out by means of a reflectometric technique using the Agilent 8753ES Network Analyser.

\section{Which dynamic process below $T_{g}$ is responding to terahertz time domain spectroscopy?}

For a fundamental understanding of the THz-TDS data of the polyalcohols, it is vital to identify the dynamic process or processes probed at the terahertz level to exhibit the changes of $\varepsilon^{\prime \prime}(v)$ at $T_{g}$ and below $T_{g}$. The answer can be found by examining the dielectric spectra obtained at these temperatures. Usually the measurement frequencies are orders of magnitude lower than terahertz. Notwithstanding, by extrapolating the observation to terahertz, the dynamic process probed at $1 \mathrm{THz}$ and showing the change can be deduced. At these low temperatures as shown by dielectric loss data at frequencies below $10^{6} \mathrm{~Hz}$ in various molecular glassformers, ${ }^{45-47}$ including the polyalcohols, the process probed is the NCL of caged dynamics. This can be verified from the figures presented later on in this paper as well as the data published by Nakanishi, and Nozaki ${ }^{63}$ from $40 \mathrm{~Hz}$ to $10 \mathrm{GHz}$ by dielectric spectroscopy of these polyalcohols. Direct observations of the NCL in the time domain from ps to ns range (note that $1 \mathrm{ps}$ corresponds to $0.16 \mathrm{THz}$ ) by optically heterodyne detected optical Kerr effect (OHD-OKE) experiments of a variety of small molecular glass-formers including ortho-terphenyl, salol, benzophenone and others at temperatures above $T_{g},{ }^{64-67}$ and certainly will continue to be observed in the glassy state. The light scattering spectra of polyisobutylene, poly(methyl methacrylate), and glycerol measured in the gigahertz frequency range all show the NCL up to $3 \mathrm{GHz}$ at temperatures below the glass transition ${ }^{17}$. All these observations higher than $1 \mathrm{GHz}$ indicate the presence of the NCL at higher frequencies up to 
$1 \mathrm{THz}$ where it is superposed on top of the microscopic peak, which is almost independent of temperature ${ }^{68}$. Thus the observed increase of the dielectric losses, $\varepsilon^{\prime \prime}(v)$ at $v=0.6$ and $1 \mathrm{THz}$, with temperature and the break at $T_{\beta}$ below $T_{g}$ is contributed by the NCL.

To proceed further we consider the plots of the $\alpha$ - and JG $\beta$-relaxation times, $\tau_{\alpha}$ and $\tau_{J G}$, against $T_{g} / T$ of the four polyalcohols adapted from the data of Do $\beta$ et al ${ }^{69,70}$ and additional data obtained by us in this study in Fig.1. Defined as the temperature at which $\tau_{\alpha}\left(T_{g}\right)=100 \mathrm{~s}$, $T_{g}$ is equal to $185 \mathrm{~K}$ for glycerol, $224 \mathrm{~K}$ for threitol, $248 \mathrm{~K}$ for xylitol, and $268 \mathrm{~K}$ for sorbitol. The Arrhenius temperature dependences of $\tau_{J G}$ have been extrapolated to lower scaled reciprocal temperatures to reach $100 \mathrm{~s}$ at $T_{g} / T_{\beta}$. In the case of glycerol, only one point was identified for $\tau_{J G}$ and the activation energy was obtained by Do $\beta$ et al. from extrapolation of the values of the series of polyalcohols. Nevertheless, these values are in good agreement with $\tau_{J G}$ of glycerol, as estimated near $T_{g}$ from an aging experiment ${ }^{71}$. The value of $T_{g} / T_{\beta}$ for sorbitol, xylitol, threitol, and glycerol are $1.60,1.55,1.36$, and 1.17 respectively, with the corresponding values of $0.63,0.65,0.74$, and 0.85 for $T_{\beta} / T_{g}$, and $169 \mathrm{~K}, 160 \mathrm{~K}, 165 \mathrm{~K}$, and $157 \mathrm{~K}$ for $T_{\beta}$. As shown before ${ }^{39}$, there is approximate agreement between $\tau_{J G}$ and the primitive relaxation time $\tau_{0}$ calculated by the $\mathrm{CM}$ equation,

$$
\tau_{0}=\left(\tau_{\alpha}\right)^{1-n}\left(t_{c}\right)^{n} \approx \tau_{J G}
$$

with $n$ from Eq.(1) and $t_{c}=1-2$ ps for molecular glass-formers and for all four polyalcohols.

The values of $T_{\beta} / T_{g}=0.63$ and 0.65 for sorbitol and xylitol respectively are effectively coincident with the value of 0.65 determined by Sibik et al. by fitting the sub- $T_{g}$ change of $T$ dependence of $\varepsilon^{\prime \prime}(v)$ at $1 \mathrm{THz}$ by two lines. The sub-T $T_{g}$ change of threitol is much weaker. 
Although Sibik et al. suggested the same value of 0.65 , but the location of the change is also consistent with $T_{\beta} / T_{g}=0.74$. In the case of glycerol, Sibik et al. apparently was looking for the change near $0.65 T_{g}$ because they considered the thermal changes in the losses originates from a universal change in the hydrogen bonding structure of the samples. Not found in glycerol was rationalized by it remaining unobservable in that case. However, the value of $T_{\beta} / T_{g}=0.85$ for glycerol determined in Fig. 1 seems to correspond to a change of $\varepsilon^{\prime \prime}$ approximately at this value, as shown by Fig.2 where the $\varepsilon^{\prime \prime}(v=1 \mathrm{THz})$ and $\varepsilon^{\prime \prime}(v=0.6 \mathrm{THz})$ data of glycerol are reproduced. The major result so far in this section is coincidence of the vitrification temperature $T_{\beta}$ of the JG $\beta$-relaxation with the temperature at which the sub- $T_{g}$ change in $\varepsilon^{\prime \prime}(v)$ of all four polyalcohols were found.

The results given above indicate that the kinetic glass-like transition of the JG $\beta$ relaxation is reflected in the dynamic process responsible for the $\varepsilon^{\prime \prime}(v)$ observed in $\mathrm{THz}$ region. But definitely the dynamic process is not the JG $\beta$-relaxation itself because its frequency $v_{J G}$ at $T_{\beta}$ is nearly 15 decades lower than $1 \mathrm{THz}$. To identify the dynamic process involved, we can get some hint of it from the dielectric relaxation spectra of the polyalcohols at temperatures below and above their respective $T_{\beta}$. Fig.3 shows our own isothermal loss spectra of sorbitol at selected temperatures, 160, 164, 168, $176 \mathrm{~K}, 183 \mathrm{~K}$ in the neighborhood centered at $T_{\beta}=169 \mathrm{~K}$. In addition, data at higher frequencies and higher temperatures from this study and from the literature $23,59,60,63,72$ are shown. Over many decade of frequencies up to $10^{5} \mathrm{~Hz}$ and beyond, $\varepsilon^{\prime \prime}(v)$ has the power law temperature dependence of $v^{-0.13}$ and weak temperature, which are the characteristics of the nearly constant loss (NCL) of the caged molecules found in many glassformers $37,39,45,46,62,63$. The dynamics of caged molecules is 
neither relaxation nor diffusion, and has no characteristic time constant. The NCL with power law frequency dependence extends from the onset frequency of the order of the microscopic excitations frequency in the infrared region ${ }^{68,72}$, and persists indefinitely to lower frequencies until it is terminated at the cut-off frequency $v_{\mathrm{NCL}}(T)$ by onset of the primitive relaxation. Thus the power law seen in Fig. 3 will continue to hold when extended to higher frequencies, and at the $\mathrm{THz}$ regime it is superposed on top of the microscopic peak, which is almost independent of temperature ${ }^{68}$. The NCL is thus the dynamic process responsible for the $\varepsilon^{\prime \prime}(v)$ at $1 \mathrm{THz}$ and change of temperatures dependence near $T_{\beta}$ for sorbitol. The same discussion can be applied also to the data of the other polyalcohols. In particular it can rationalize the results of Fig. 2 for glycerol, where $\varepsilon^{\prime \prime}(v=0.6 \mathrm{THz})$ exhibits a stronger temperature change than $\varepsilon^{\prime \prime}(v=1 \mathrm{THz})$

The lower bound of the NCL regime, $\nu_{\mathrm{NCL}}(T)$, is higher than the primitive relaxation frequency, $v_{0}(T) \approx v_{\mathrm{JG}}(T)$, but normally the former is higher than the latter by one or two decades at temperatures above $T_{g}$ as can be seen from examples shown in $^{37,39,44,45,46}$ and also from some spectra in Fig.3. This relation between $v_{\mathrm{NCL}}(T)$ and $v_{0}(T) \approx v_{\mathrm{JG}}(T)$ holds in the spectra of xylitol (see Fig.4), threitol, and glycerol (see Figs.S1 and S2 in the Supporting Information), justifying that the primitive/JG- $\beta$ relaxation is the terminator of the caged dynamics regime ${ }^{41}$. Again, the dielectric $\varepsilon^{\prime \prime}(v)$ data of these three polyalcohols exhibit the NCL starting at frequencies about 10 decades below $1 \mathrm{THz}$ at temperatures above their respective $T_{\beta}$, and is expected to remain as NCL for all temperatures below. In Fig.S3 (see Supporting Information) the weak temperature dependence of the NCL proportional to $\exp \left(T / T_{\mathrm{o}}\right)$ in a range of temperatures around $T_{\beta}=157 \mathrm{~K}$ of glycerol is shown. Therefore, by 
these figures, the NCL is identified as the dynamic process contributing to the $\varepsilon^{\prime \prime}(v)$ at $1 \mathrm{THz}$ and giving rise to the change of temperatures dependence near $T_{\beta}$ for these other polyalcohols. Naturally the question following this identification is why the NCL is sensitive to the vitrification of the JG $\beta$-relaxation? The answer to this question will be given later, and before that present in the next section are dielectric measurements of two pharmaceutical glassformer, flufenamic acid and indomethacin to determine the $\alpha$ - and the JG $\beta$-relaxation times, $T_{\beta}$ and the ratio $T_{\beta} / T_{g}$ for comparison with THz-TDS data when it becomes available in the open literature.

\section{Dielectric relaxation data of flufenamic acid and indomethacin}

The isothermal dielectric loss spectra of flufenamic acid are shown in Fig.5. The prominent $\alpha-$ loss peak is fitted by the Fourier transform of the Kohlrausch function with $n=0.44$ shown by example at one temperature. At higher frequencies is a well resolved secondary relaxation loss peak. There is approximate agreement between the secondary relaxation frequency $v_{\mathrm{JG}}(T)$ and the primitive relaxation frequency $v_{0}(T)$ calculated by Eq.(2). This satisfies one criterion to indicate that the observed secondary relaxation is the JG $\beta$-relaxation. Against reciprocal temperature, the plots of logarithm of $\tau_{\alpha}(T)$ and $\tau_{\mathrm{JG}}(T)$ in Fig.6 enable determination of the dielectric $T_{g}=261 \mathrm{~K}$ when $\tau_{\alpha}(T)=100 \mathrm{~s}$ and extrapolation of the Arrhenius dependence of $\tau_{\mathrm{JG}}(T)$ down to $T_{\beta}=189 \mathrm{~K}$ at which $\tau_{\mathrm{JG}}(T)=100 \mathrm{~s}$. Thus $T_{\beta} / T_{g}=0.67$ in the case of flufenamic

acid, and this value is predicted at or near which the $\varepsilon^{\prime \prime}(v)$ observed by THz-TDS at $1 \mathrm{THz}$ will change temperature dependence ${ }^{61}$.

On cooling the sample much further below $T_{g}$ to move the $\alpha$ - and the $\beta$-relaxation out of the instrument frequency window, the NCL appears at higher frequencies and lower 
temperatures in the loss spectra (Fig.5). The $\varepsilon^{\prime \prime}(v)$ data at $T_{\beta}=189 \mathrm{~K}$ and below show clear power law dependence $v^{-\lambda}$ with $\lambda$ of the order of 0.1 and weak temperature dependence typical of the NCL. The $v^{-\lambda}$-dependence seen by conventional dielectric measurements at frequencies lower than $10^{5} \mathrm{~Hz}$ should persists up to the terahertz region, and the dynamics probed by THz-TDS is the NCL of caged molecules at temperatures below and above $T_{\beta}=189$.

In contrast to flufenamic acid, the JG $\beta$-relaxation of indomethacin has very small dielectric strength and is not easily resolved in isothermal dielectric spectra ${ }^{73-76}$. However its presence has been detected by either applying elevated pressure or by first compressing a sample in the liquid state followed by cooling the sample below $T_{\mathrm{g}}$ and releasing the pressure $^{74}$. An extrapolation of the suggested Arrhenius temperature dependence of $\tau_{\mathrm{JG}}(T)$ down to $100 \mathrm{~s}$ determines ${ }^{73} T_{\beta}=184.5 \mathrm{~K}$ at which $\tau_{\mathrm{JG}}(T)=100 \mathrm{~s}$. This together with $T_{g}=316.25$ $\mathrm{K}$ gives $T_{\beta} / T_{g}=0.58$. There is a large uncertainty in this value because of the long extrapolation from values of $\tau_{\mathrm{JG}}(T)$ at two temperatures each with large uncertainty. Another way to determine $T_{\beta}$ is to use the calculated value of $\tau_{0}\left(T_{g}\right)=2.42 \times 10^{-4} \mathrm{~s}$ by Eq.(2) with $n=0.41$ and $\tau_{\alpha}\left(T_{g}\right)=100 \mathrm{~s}$ and $T_{g}=316.25 \mathrm{~K}$, and the fact that $\tau_{0}\left(T_{g}\right) \approx \tau_{\beta}\left(T_{g}\right)$. The same value of $n=0.41$ was obtained by both Carpentier et al. $^{73}$ and Wojnarowska et al. ${ }^{74}$ By manipulating the differential scanning calorimetry (DSC) technique, Vyazovkin and Dranca ${ }^{76}$ were able to detect the $\beta$-relaxation in the glassy state of indomethacin and found that its activation energy $E_{\beta}$ is $56 \mathrm{~kJ} / \mathrm{mol}$. Combining this value of $E_{\beta}$ with $\tau_{0}\left(T_{g}\right)=2.42 \times 10^{-4} \mathrm{~s}$, the Arrhenius dependence of $\tau_{\mathrm{JG}}(T) \approx \tau_{0}(T)$ is given by $1.35 \times 10^{-13} \exp (6736 / T)$. Hence another estimate of $T_{\beta}$ is equal to $196.5 \mathrm{~K}$ by the definition of $\tau_{\mathrm{JG}}\left(T_{\beta}\right) \approx \tau_{0}\left(T_{\beta}\right)=100 \mathrm{~s}$, and $T_{\beta} / T_{g}=0.62$. The two predicted values of $T_{\beta} / T_{g}$ can be compared with the $\mathrm{THz}$ data when published. It is worth 
pointing out that present in the glassy state of indomethacin is the well resolved and intense $\gamma$ relaxation. Faster than the JG $\beta$-relaxation with Arrhenius activation energy $E_{\gamma}=38 \mathrm{~kJ} / \mathrm{mol}$, the $\gamma$-relaxation is pressure-insensitive and intramolecular in origin because it is pressureinsensitive $^{74}$. The NCL is eclipsed by the $\gamma$-relaxation and cannot be observed directly in the low frequency dielectric spectra.

\section{Relation of the NCL with the primitive/JG $\beta$ relaxation}

In the $\mathrm{CM}$, the dynamics evolve and change with time $\mathrm{e}^{37,39,41-44}$. Starting at short times is the motion of molecules confined within cages via the intermolecular potential, and the loss is determined by anharmonicity of the potential. The caged dynamics is not a normal relaxation process. It has no characteristic time, and hence the loss is a power law, $\chi^{\prime \prime}(v)=B(T) v^{-\lambda}$ with $\lambda<<1$, or appropriately referred to as the nearly constant loss (NCL) in the susceptibility spectrum. Neither cooperativity nor heterogeneity applies to the NCL of caged dynamics because molecules remain caged throughout the regime, and the loss originates from the anharmonicity of the potential. Although caged dynamics form the core issue of the idealized Mode Coupling Theory ${ }^{66,67,78}$ but the NCL is not a prediction of $i^{16,17,28}$. This NCL regime persists until the cages are dissolved by the onset of the primitive relaxation of the $\mathrm{CM}^{37,39,41-}$ ${ }^{44}$, the motion of which involving the entire molecule (i.e. a generalization and analogue of the totally rigid molecules studied by Johari and Goldstein ${ }^{79}$. Thus, the primitive relaxation time, $\tau_{0}(T, P)$, is an order-of-magnitude upper bound of the NCL time regime, a prediction verified by experimental data and molecular dynamics simulations in many molecular glassformers ${ }^{37-}$ ${ }^{46}$, and ionic conductors ${ }^{47-51,57-59}$. Application of high electric fields in dielectric spectroscopy has the effect of increased loss from all genuine relaxation processes including the JG $\beta$ relaxation starting from the primitive relaxation in glycerol and sorbitol ${ }^{80-82}$. The cause of the 
increased loss originates from the heterogeneous dynamics of the relaxation processes. However no effect of the high field was found in the NCL in these high field experiments, as pointed out in a more recent paper ${ }^{44}$. The analyses of these experimental finding also provides additional evidence that $v_{0}$ is an lower bound of the NCL frequency regime.

The NCL is terminated when it reaches the critical value, $\varepsilon_{\text {max }}^{\prime \prime}$, at the cut-off frequency $v_{\mathrm{c}}(T)$ equal to $v_{0}$ in order of magnitude, by the onset of the primitive relaxation. The magnitude of $\varepsilon_{\text {max }}^{\prime \prime}$ is independent of the value of $v_{\mathrm{c}}(T)$ or $v_{0}(T)$ because the same criterion for the termination of the NCL applies. Since from its onset frequency, $v_{\mathrm{on}}$, to $v_{\mathrm{c}}(T)$, the $\varepsilon^{\prime \prime}(v)$ of the NCL increase by the same amount, it follows from the power law dependence of $\varepsilon^{\prime \prime}(v)=B(T) v^{-\lambda}$ that at any intermediate frequency $v$ we have the proportionality relation for the magnitude of the NCL,

$$
N C L(v) \propto \varepsilon_{\text {max }}^{\prime \prime} /\left[\log v_{o n}-\log v_{c}(T)\right]
$$

On heating, the value of $v_{\mathrm{c}}(T)$ increases following $v_{0}(T) \approx v_{\mathrm{JG}}(T)$, and the denominator in relation (3) decreases and hence $N C L(v)$ increases. This monotonic increase of $N C L(v)$ with temperature is commonly observed in dielectric ${ }^{23,37,39,45,46}$ and light scattering ${ }^{16,17}$ studies, and also in THz-TDS ${ }^{60,61}$. Moreover, as consequence of the vitrification of the $\beta$-relaxation (i.e. the secondary glass transition ${ }^{83}$ ) on crossing $T_{\beta}$, the change of $v_{0}(T) \approx v_{\mathrm{JG}}(T)$ with temperature above $T_{\beta}$ is stronger than below $T_{\beta}$. This change of $T$-dependence of $v_{0}(T) \approx v_{\mathrm{JG}}(T)$ and as well the cut-off frequency $v_{\mathrm{c}}(T)$ at $T_{\beta}$ gives rise to a corresponding change of $T$-dependence, as can be inferred from relation (3). The weak temperature dependence of the intensity factor, $A(T) \propto \exp \left(T / T_{0}\right)$, where $T<T_{0}$ is also derivable from the primitive relaxation frequency, 
$v_{0}(T, P) \equiv 2 \pi / \tau_{0}(T, P)$, acting as the lower bound of the NCL frequency regime ${ }^{49}$. Although the derivation in Ref.[49] is for caged ions of ionically conducting systems, it is applicable also to cage molecules in glass-formers.

Even better known from experiments ${ }^{43,52-54}$, and theoretically from Eq.(2) is the change of the temperature dependence of $v_{0}(T) \approx v_{\mathrm{JG}}(T)$ at $T_{g}$ from the Arrhenius dependence below to a stronger dependence above. The same relation (3) explains the corresponding change in temperature dependence of $N C L(v)$ on crossing $T_{g}$, which was observed in neutron scattering ${ }^{2-}$ ${ }^{11,34}$, and by dynamic light scattering ${ }^{16,42}$. In closing this section we mention other studies by simulations of the fast dynamics in model glassformers ${ }^{84-86}$ and from experiment ${ }^{87}$ offering different interpretations than ours. Mainly focused on the relation between cooperative motions, elasticity and free volume, the authors of these studies did not take into account the contribution of the JG relaxation, and thus no prediction or explanation was provided for the change of fast dynamics at $T_{\beta}$.

\section{Summary and Conclusion}

The isothermal and isochronal dielectric relaxation data of the polyalcohols we presented have identified the nearly constant losses in susceptibility spectra of caged molecules are the process probed by THz-TDS in the study by Sibik et al. ${ }^{60,61}$. Therefore the NCL is the process responsible for the observed change in the temperature dependence of $\varepsilon^{\prime \prime}(v)$ at $1 \mathrm{THz}$ in the experiment. From the relaxation spectra of the polyalcohols we determine the structural $\alpha$ relaxation time $\tau_{\alpha}$, and the JG $\beta$ relaxation time $\tau_{J G}$. The nominal glass transition temperature $T_{g}$ and the "secondary glass transition" temperature $T_{\beta}$ are determined as the temperatures at which $\tau_{\alpha}$ and $\tau_{J G}$ reach 100 s respectively. 
The crux of the paper is Coupling Model description of the evolution of dynamics with time. The dynamics start from the caged molecules regime, which is terminated by the primitive relaxation involving rotation and/or translation of individual molecules. In turn, the primitive relaxation is the start or precursor of the evolution of the relaxation dynamics with time that involves the participation of increasing number of molecules. These spatially and dynamically heterogeneous relaxation processes, collectively considered as the JG $\beta$ relaxation, is spatially and dynamically heterogeneous as well as to some extent cooperative. These properties of the JG $\beta$-relaxation explain why it shows up at some temperature $T_{\beta}$ below $T_{g}$ in heat capacity measurements by various techniques of calorimetry ${ }^{77,88-91}$, and positronium annihilation spectroscopy ${ }^{83,92}$, and justify calling it the secondary glass transition consistent with the $T \rho^{-\gamma}$-dependence of $\tau_{J G}{ }^{57}$ Furthermore, the freezing of the motions related to $\mathrm{JG} \beta$-relaxation has been recently $\operatorname{shown}^{93}$ to contribute to glass entropy and enthalpy. These findings imply that glasses have a non-equilibrium structure characterized by two fictive temperatures ${ }^{93}$ : the highest $T_{f}$ (close to $T_{g}$ ), that corresponds to the kinetic freezing of $\alpha$ - cooperative motions and the lower $T_{f, J G}$ (close to $T_{\beta}$ ) that corresponds to kinetic freezing of local fluctuations. Based on these experimental facts and theoretical considerations, the occurrence of secondary glass transition of the JG $\beta$-relaxation at $T_{\beta}$ is natural. The temperature dependence of $\tau_{J G}$ changes from the stronger Arrhenius dependence above $T_{\beta}$ to a weaker one below $T_{\beta}$. The final stage of the evolution of dynamics is the heterogeneous and cooperative $\alpha$-relaxation having time correlation function given by the Kohlrausch function in eq.(1), and its relaxation time $\tau_{\alpha}$ is connected to $\tau_{0} \approx \tau_{J G}$ by eq.(2) which also has been amply verified in many glassformers and mixtures by experiments. 
The termination of caged molecules dynamics regime by the onset of the primitive relaxation acting as the precursor of the $\mathrm{JG} \beta$ relaxation is the origin of the connection of the caged molecules dynamics with relaxation processes. The cage molecules dynamics manifested in susceptibility as the nearly constant loss (NCL) is thus related in its magnitude to the relaxation frequencies $v_{0}(T) \approx v_{\mathrm{JG}}(T)$. The well understood changes of temperature dependence of $v_{0}(T) \approx v_{\mathrm{JG}}(T)$ at $T_{g}$ and $T_{\beta}$ are transferred to the corresponding changes of the NCL. Density changes on crossing $T_{g}$ and $T_{\beta}$ also can independently rationalize the changes of the $T$-dependence of NCL. Through the steps summarized in the above we have a microscopic explanation of the sub- $T_{g}$ change of $\varepsilon^{\prime \prime}(v)$ data at $T_{T H z}$ observed in the polyalcohols by THz-TDS. In this explanation, the sub- $T_{g}$ change of $\varepsilon^{\prime \prime}(v)$ data should occur at $T_{\beta}$ obtained from the analysis of conventional dielectric spectroscopy. The good agreements of $T_{\beta}$ with $T_{T H z}$ in the polyalcohols are strong support of the explanation. Analysis of the dielectric spectra of flufenamic acid and indomethacin gives the values of $T_{\beta}$ for these two pharmaceutical glass-formers. The prediction that $T_{\beta}$ should be approximately equal to $T_{T H z}$ can be checked against THz-TDS data of these glass-formers ${ }^{61}$ when published in the future.

Although we focus our attention on data from THz-TDS data, other high frequency/short time techniques such as dynamic light scattering and neutron scattering will serve the same purpose of showing the presence of the sub- $T_{g}$ change originating from the NCL of caged dynamics. As an example we cite the neutron scattering data of a cis/trans mixture of decalin by Plazanet and Schober $^{8}$. The mean square displacements from elastic intensity measurements show a change of temperature dependence at $T_{n} \approx 90 \mathrm{~K}$ and at $T_{g}=137$ $\mathrm{K}$. The ratio $T_{n} / T_{g}=0.66$. If the low frequency susceptibility spectra of decalin are available to 
deduce the vitrification temperature of the primitive or the JG $\beta$-relaxation, $T_{\beta}$, it can be compared with $T_{n}$. In the case of decalin the dielectric strength is too weak, and the $\beta$ relaxation has not been detected ${ }^{94}$. Notwithstanding, there are other glass-formers for which the sub- $T_{g}$ change was observed at $T_{n}$ by other high frequency techniques other than THzTDS, and to be compared with $T_{\beta}$ which also can be determined by low frequency dielectric spectroscopy and calorimetry. These will be the subject of a follow-up paper.

As a final remark, this paper should not be considered as just an explanation of the data from THz-TDS. Rather it serves as another demonstration of the link between the caged molecules dynamics and the primitive relaxation, the precursor of the JG $\beta$-relaxation, showing up before in many other experimental investigations.

\section{Acknowledgments}

We thank Juraj Sibik for stimulating discussion of his experimental data by THz-TDS. This study has been supported by Italian Foreign Affairs Ministry - DGPSP within the Executive Programme for Scientific and Technological Cooperation between the Italian Republic and the People’s Republic of China 2013-2015 (Significant Research Project PGR00137).

\section{Supporting Information Available}

More details on the dielectric loss spectra of polyalcohols are provided in the Supporting Information. This information is available free of charge via the Internet at http://pubs.acs.org 


\section{References}

${ }^{1}$ Knaak, W.; Mezei, F.; Farago, B.; Observation of Scaling Behaviour of Dynamic Correlations near Liquid-Glass Transition, Europhys. Lett. 1988, 7, 529.

${ }^{2}$ Petry, W.; Bartsch, E.; Fujara, F.; Kiebel, M.; Sillescu, H.; Farago, B.; Dynamic Anomaly in the Glass Transition Region of Orthoterphenyl, Z. Phys. B 1991, 83, 175.

${ }^{3}$ Frick, B.; Farago, B.; Richter, D.; Temperature Dependence of the Nonergodicity Parameter in Polybutadiene in the Neighborhood of the Glass Transition, Phys. Rev. Lett., 1990, 64, 2921.

${ }^{4}$ Mezei, F.; Russina, M.; Intermediate Range Order Dynamics Near the Glass Transition, $J$. Phys.: Condens. Matter 1999, 11, A341.

${ }^{5}$ Arbe, A.; Buchenau, U.; Willner, L.; Richter, D.; Colmenero, J.; Study of the Dynamic Structure Factor in the $\beta$ Relaxation Regime of Polybutadiene, Phys. Rev. Lett. 1996, 76, 1872.

${ }^{6}$ Frick, B.; Richter, D.; Petry, W.; Buchenau, U.; Study of the Glass Transition Order Parameter in Amorphous Polybutadiene by Incoherent Neutron Scattering, Z. Phys. B. Condens. Matter 1989, 70, 73-79.

${ }^{7}$ Mermet, A.; Duval, E.; Surovtsev, N.V.; Jal, J.F.; Dianoux, A.J., Yee, A.F., Localized Fast Relaxation in Poly(methyl methacrylate) Glass, Europhys. Lett. 1997, 38, 515-520

${ }^{8}$ Plazanet, M.; Schober, H.; Anharmonicity in a Fragile Glass-Former Probed by Inelastic Neutron Scattering, Phys. Chem. Chem. Phys., 2008, 10, 5723-5729.

${ }^{9}$ Inoue, R., Kanaya, T.; Heterogeneous Dynamics of Polymer Thin Films as Studied by Neutron Scattering, Adv Polym Sci, 2013, 252, 107-140.

${ }^{10}$ Buchenu, U.; Zorn, R.; Ramos, M.A., Probing Cooperative Liquid Dynamics with the Mean Square Displacement, Phys.Rev.E, 2014, 90, 042312.

${ }^{11}$ Ngai, K.L. ; Habasaki, J. An Alternative Explanation of the Change in T-Dependence of the Effective Debye-Waller Factor at Tc or Tb, J. Chem. Phys. 2014, 141, 114502.

${ }^{12}$ Monaco, G. ; Fioretto, D.; Masciovecchio, C.; Ruocco, G.; Sette, F.; Fast Relaxational Dynamics in the $o$-Terphenyl Glass, Phys. Rev. Lett. 1999, 82,1776.

${ }^{13}$ Li, G.; Du, W. M. ; Hernandez, J.; Cummins, H. Z.; Locating the Nonergodicity-Parameter Anomaly near the Liquid-to-Glass Crossover Temperature in CaKNO3 by Brillouin Scattering, Phys. Rev. E, 1993, 48, 1192. 
${ }^{14}$ Steffen, W.; Patkowski, A.; Glaser, H.; Meier, G.; Fischer, E. W.; Depolarized-LightScattering Study of Orthoterphenyl and Comparison with the Mode-Coupling Model, Phys. Rev. B, 1994, 49, 2992.

${ }^{15}$ Gapinski, J.; Steffen, W.; Patkowski, A. ; Sokolov, A. P.; Kisliuk, A.; Buchenau, U.; Russina, M.; Mezei, F.; Schober, H.; Spectrum of Fast Dynamics in Glass Forming Liquids: Does the "Knee" Exist?, J. Chem. Phys., 1999, 110, 2312-2315.

${ }^{16}$ Sokolov, A. P.; Kisliuk, A.; Novikov, V. N.; Ngai, K.L.; Observation of Constant Loss in Fast Relaxation Spectra of Polymers, Phys. Rev. B 2001, 63, 172204.

${ }^{17}$ Kisliuk, A., Novikov, V. N.;Sokolov, A. P.; Constant Loss in Brillouin Spectra of Polymers,J. Polym. Sci., Part B: Polym. Phys., 2002, 40, 201.

${ }^{18}$ Sette, F.; Krisch, M. H.; Masciovecchio, C.; Ruocco, G.; Monaco, G.; Dynamics of Glasses and Glass-Forming Liquids Studied by Inelastic X-ray Scattering. Science 1998, 280, 15501555 .

${ }^{19}$ Scopigno, T.; Ruocco, G.; Sette, F.; Monaco, G.; Is the Fragility of a Liquid Embedded in the Properties of Its Glass? Science 2003, 302, 849-852.

${ }^{20}$ Comez, L.; Corezzi, S.; Monaco, G.; Verbeni, R. ; Fioretto, D.; Ergodic to Nonergodic Transition in Liquids with a Local Order: The Case of m-Toluidine, Phys. Rev. Lett. 2005, 94, 155702 .

${ }^{21}$ Lunkenheimer, P. ; Pimenov, A.; Loidl, A.; Fast Dynamics in CKN and CRN Investigated by Dielectric Spectroscopy, Phys. Rev. Lett. 1997, 78, 2995.

${ }^{22}$ Lunkenheimer, P.; Pimenov, A.; Dressel, M.; Goncharov, Y.; Böhmer, R.; Loidl, A., Fast Dynamics of Glass-Forming Glycerol Studied by Dielectric Spectroscopy. Phys. Rev. Lett. 1996, 77, 318-321.

${ }^{23}$ Kastner, S. ; Köhler, M.; Goncharov, Y.; Lunkenheimer, P. ; Loidl, A.; High-Frequency Dynamics of Type B Glass Formers Investigated by Broadband Dielectric Spectroscopy, J.Non-Cryst. Solids, 2011, 357, 510-514.

${ }^{24}$ Yomogida, Y.; Sato, Y.; Nozaki, R.; Mishina, T. Comparative Dielectric Study of Monohydric Alcohols with Terahertz Time-Domain Spectroscopy. J. Mol. Struct. 2010, 981, $173-178$.

${ }^{25}$ Miao, Q.; Tian, L.; Zhao, K.; Zhou, Q.; Shi, Y.; Zhao, D.; Zhao, S.; Zhang, C. Detection and Identification of Selected Alcohols Using Terahertz Time-domain Spectroscopy. J. Phys.

(Paris) 2011, 012220. 
${ }^{26}$ Sibik, J.; Shalaev, E. Y.; Zeitler, J. A. Glassy Dynamics of Sorbitol Solutions at Terahertz Frequencies. Phys. Chem. Chem. Phys. 2013, 15, 11931-11942.

${ }^{27}$ Wietzke, S.; Jansen, C.; Reuter, M.; Jung, T.; Kraft, D.; Chatterjee, S.; Fischer, B.M.; Koch, M.; , Terahertz Spectroscopy on Polymers: A Review of Morphological Studies, J. Mol. Struct. 2011, 1006, 41-51.

${ }^{28}$ Cang, H., Novikov, V.N., Fayer, M.D., Logarithmic Decay of the Orientational Correlation Function in Supercooled Liquids on the ps to ns Time Scale, J. Chem. Phys. 2003, 118, 28002807.

${ }^{29}$ Donati, C.; Glotzer, S.C.; Poole, P.H.; Kob, W.; Plimpton, S.J., Spatial Correlations of Mobility and Immobility in a Glass-Forming Lennard-Jones Liquid, Phys. Rev. E 1999, 60, 3107.

${ }^{30}$ Bordat, P.; Affouard, F.; Descamps, M.; Ngai, K.L.; Does the Interaction Potential Determine Both the Fragility of a Liquid and the Vibrational Properties of its Glassy State? Phys. Rev. Lett. 2004, 93(10):105502.

${ }^{31}$ Habasaki, J.;Ngai, K.L.; Molecular Dynamics Simulation of Ion Dynamics in Glassy Ionic Conductors: Evidence of the Primitive Ion Hopping Process, J. Non-Cryst. Solids 2006, 352, $5170-5177$.

${ }^{32}$ Coslovich, D.; Roland, C. M.; Density Scaling in Viscous Liquids: From Relaxation Times to Four-Point Susceptibilities, J. Chem. Phys. 2009, 131, 151103.

${ }^{33}$ Roland, C. M. ; Fragiadakis, D.; Coslovich, D.; Capaccioli, S. ; Ngai, K. L.; Correlation of Nonexponentiality with Dynamic Heterogeneity From Four-Point Dynamic Susceptibility $\chi_{4}(\mathrm{t})$ and its Approximation ChiT, J. Chem. Phys. 2010, 131, 124507.

${ }^{34}$ Ngai, K.L. ; Dynamic and Thermodynamic Properties of Glass-Forming Substances, J. NonCryst. Solids 2000, 275, 7-51.

${ }^{35}$ Angell, C. A. ; Ten Questions on Glassformers, and a Real Space `Excitations' Model with Some Answers on Fragility and Phase Transitions, J. Phys.: Condens. Matter, 2000, 12, 6463.

${ }^{36}$ Angell, C. A.; Ngai, K. L.; McKenna, G. B.; McMillan, P. F.; Martin, S. W.; Relaxation in Glassforming Liquids and Amorphous Solids, J. Appl. Phys. 2000, 88, 3113.

${ }^{37}$ Ngai, K. L. ; Relaxation and Diffuson in Complex Systems, 2011 Springer (New York).

${ }^{38}$ Ngai, K. L., Relation between Some Secondary Relaxations and the Alpha Relaxations in Glass- Forming Materials According to the Coupling Model, J. Chem. Phys., 1998, 109, 6982. 
${ }^{39}$ Ngai, K.L.; An Extended Coupling Model Description of the Evolution of Dynamics with Time in Supercooled Liquids and Ionic Conductors, J. Phys.: Condens. Matter 2003, 15, S1107-S1125.

${ }^{40}$ Ngai, K. L.; Paluch,M:; Classification of Secondary Relaxation in Glass-Formers Based on Dynamic Properties, J. Chem. Phys., 2004, 120, 857.

${ }^{41}$ Ngai, K. L., in Slow Dynamics in Complex Systems: 3rd International Symposium, edited by Tokuyama M. and Oppenheim, I., AIP Conf. Proc. 2004, 708, 515.

${ }^{42}$ Ngai, K. L. ; Why the Fast Relaxation in the Picosecond to Nanosecond Time Range Can Sense the Glass Transition, Philos. Mag. 2004, 84, 1341.

${ }^{43}$ Capaccioli, S.; Paluch, M.; Prevosto, D.; Wang, L.-M.;Ngai, K. L. Many-Body Nature of Relaxation Processes in Glass-Forming Systems, J. Phys. Chem. Lett. 2012, 3, 735.

${ }^{44}$ Ngai, K.L. Interpreting the Nonlinear Dielectric Response of Glass-formers in Terms of the Coupling Model, J. Chem.Phys. 2015, 142, 114502.

${ }^{45}$ Capaccioli, S. ; Thayyil, M. S.; Ngai, K. L. Critical Issues of Current Research on the Dynamics Leading to Glass Transition, J. Phys. Chem. B 2008, 112, 16035.

${ }^{46}$ Ngai, K.L.; Paluch, M. Inference of the Evolution from Caged Dynamics to Cooperative Relaxation in Glass-Formers from Dielectric Relaxation Data, J. Phys. Chem. B 2003, 107, 6865-6872.

${ }^{47}$ León, C. ; Rivera, A.; Várez, A.; Sanz, J.; Santamaria, J.; Ngai, K.L. Origin of Constant Loss in Ionic Conductors, Phys. Rev. Lett. 2001, 86, 1279.

${ }^{48}$ Ngai, K. L.; León, C. Cage Decay, Near Constant Loss, and Crossover to Cooperative Ion Motion in Ionic Conductors: Insight from Experimental Data, Phys. Rev. B, 2002, 66, 064308.

${ }^{49}$ Ngai, K.L.; Habasaki, J.; Hiwatari, Y.; León, C. A Combined Molecular Dynamics Simulation, Experimental and Coupling Model Study of the Ion Dynamics in Glassy Ionic Conductors, J. Phys. Condens. Matter 2003, 15, S1607.

${ }^{50}$ Rivera, A., León, C. ; Sanz, J.; Santamaria, J. ; Moynihan, C. T.; Ngai, K. L. Crossover from Ionic Hopping to Nearly Constant Loss in the Fast Ionic Conductor Li0.18La0.61TiO3, Phys. Rev. B 2002, 65, 224302.

${ }^{51}$ Ngai, K. L. ; Habasaki, J.; Leon, C.; Rivera, A. Comparison of Dynamics of Ions in Ionically Conducting Materials and Dynamics of Glass-Forming Substances: Remarkable Similarities, Z. Phys. Chem., 2005, 21947-70. 
${ }^{52}$ Paluch, M. ; Roland, C.M.; Pawlus, S.; Zioło, J.; Ngai, K. L. Does the Arrhenius Temperature Dependence of the Johari-Goldstein Relaxation Persist above Tg? Phys. Rev. Lett. 2003, 91, 115701.

${ }^{53}$ Mierzwa, M. ; Pawlus, S.; Paluch, M.; Kaminska, E.; Ngai, K. L. Correlation between Primary and Secondary Johari-Goldstein Relaxations in Supercooled Liquids: Invariance to Changes in Thermodynamic Conditions, J. Chem. Phys. 2008, 128, 044512.

${ }^{54}$ Kessairi, K.; Capaccioli, S.; Prevosto, D. ; Lucchesi, M. ; Sharifi, S.; Rolla, P. A. Interdependence of Primary and Johari-Goldstein Secondary Relaxations in Glass-Forming Systems, J. Phys. Chem. B 2008, 112, 4470.

${ }^{55}$ Böhmer, R. ; Diezemann, G.; Geil, B. ; Hinze, G.; Nowaczyk, A.; Winterlich, M. Correlation of Primary and Secondary Relaxations in a Supercooled Liquid, Phys. Rev. Lett. 2006, 97, 135701.

${ }^{56}$ Bedrov, D.; Smith, G. D.; Secondary Johari-Goldstein Relaxation in Linear Polymer Melts Represented by a Simple Bead-Necklace Model, J. Non-Cryst. Solids 2011, 357, 258.

${ }^{57}$ Ngai, K. L. ; Habasaki, J. ; Prevosto, D. ; Capaccioli, S. ; Paluch, M.; Thermodynamic Scaling of $\alpha$-Relaxation Time and Viscosity Stems from the Johari-Goldstein $\beta$-Relaxation or the Primitive Relaxation of the Coupling Model, J. Chem. Phys. 2012, 137, 034511.

${ }^{58}$ Jarosz, G.; Mierzwa, M.; Ziolo, J.; Paluch, M.; Shirota, H.; Ngai, K. L., Glass Transition Dynamics of Room-Temperature Ionic Liquid 1-Methyl-3-trimethylsilylmethylimidazolium Tetrafluoroborate, J. Phys. Chem. B 2011, 115, 12709-12716.

${ }^{59}$ Hensel-Bielowka, S. ; Ngai, K.L.; Swiety-Pospiech, A.; Hawelek, L.; Knapik, J.; Sawicki, W. ; Paluch, M.; On the Molecular Origin of Secondary Relaxations in Amorphous Protic Ionic Conductor Chlorpromazine Hydrochloride-High Pressure Dielectric Studies, J. NonCryst.Solids 2015, 407, 81-87.

${ }^{60}$ Sibik, J.; Elliott, S.R.; Zeitler, J.A.; Thermal Decoupling of Molecular-Relaxation Processes from the Vibrational Density of States at Terahertz Frequencies in Supercooled HydrogenBonded Liquids, J. Phys. Chem. Lett. 2014, 5, 1968-1972.

${ }^{61}$ Sibik, J.; Zeitler, J.A; XIV International Workshop on Complex Systems, Fai della Paganella, Italy, 22-25 March 2015; in press.

${ }^{62}$ Kudlik, A.; Benkhof, S.; Blochowicz, T.; Tschirwitz, C.; Rössler, E. The Dielectric Response of Simple Organic Glass Formers, J. Mol.Struct. 1999, 479, 201-218.

${ }^{63}$ Nakanishi, M.; and Nozaki, R.; High-Frequency Broadband Dielectric Spectroscopy on Sugar Alcohols Below Tg, J. Non-Cryst. Solids, 2010, 356, 733-737. 
${ }^{64}$ Gottke, S. D.; Brace, D. D.; Hinze, G.; Fayer, M. D.; Time Domain Optical Studies of Dynamics in Supercooled O-terphenyl: Comparison to Mode Coupling Theory on Fast and Slow Time Scales, J. Phys.Chem. B 2001, 105, 238.

${ }^{65}$ Brace, D. D.; Gottke, S. D.; Cang, H.; Fayer, M. D.; Orientational Dynamics of the Glass Forming Liquid, Dibutylphthalate: Time Domain Experiments and Comparison to Mode Coupling Theory, J. Chem.Phys. 2002, 116, 1598.

${ }^{66}$ Hinze, G. ; Brace, D.D.; Gottke, S.D.; Fayer, M.D.; A Detailed Test of Mode-Coupling Theory on all Time Scales: Time Domain Studies of Structural Relaxation in a Supercooled Liquid, J. Chem. Phys. 2000, 113, 3723-3733.

${ }^{67}$ Cang, H.; Novikov, V. N.; Fayer, M. D.; Logarithmic Decay of the Orientational Correlation Function in Supercooled Liquids on the ps to ns Time Scale, J. Chem. Phys. 2003, 118, 2800.

${ }^{68}$ Strom, U.; Hendrickson, J. R.; Wagner, R. J.; Taylor, P. C.; Disorder-Induced Far Infrared Absorption in Amorphous Materials. Solid State Commun. 1974, 15, 1871-1875.

${ }^{69}$ Döß, A.; Paluch, M.; Sillescu, H.; Hinze, G., From Strong to Fragile Glass Formers: Secondary Relaxation in Polyalcohols. Phys. Rev. Lett. 2002, 88, 095701;

${ }^{70}$ Döß, A.; Paluch, M.; Sillescu, H.; Hinze, G., Dynamics in Supercooled Polyalcohols: Primary and Secondary Relaxation, J. Chem. Phys., 2002, 117, 6582.

${ }^{71}$ Ngai, K.L. ; Lunkenheimer, P.; Leon, C.; Schneider, U.; Brand, R.; Loidl, A.; Nature and Properties of the Johari-Goldstein Beta-Relaxation in the Equilibrium Liquid State of a Class of Glass-Formers, J. Chem. Phys., 2001, 115, 1405-1413.

${ }^{72}$ Schneider, U.; Lunkenheimer, P.; Brand, R.; Loidl, A.; Dielectric and Far-Infrared Spectroscopy of Glycerol, J. Non-Cryst. Solids, 1998, 235-237, 173-179.

${ }^{73}$ Carpentier, L. ; Decressain, R.; Desprez, S.; Descamps, M.; Dynamics of the Amorphous and Crystalline Alpha-, Gamma-Phases of Indomethacin,J. Phys. Chem. B 2006, 110, 457.

${ }^{74}$ Wojnarowska, Z. ; Adrjanowicz, K.; Wlodarczyk, P.; Kaminska, E.; Kaminski, K.; Grzybowska, K. ; Wrzalik, R.; Paluch, M.; Ngai, K. L.; Broadband Dielectric Relaxation Study at Ambient and Elevated Pressure of Molecular Dynamics of Pharmaceutical: Indomethacin, J. Phys. Chem. B 2009, 113, 12536.

${ }^{75}$ Correia, N.T.; Moura Ramos, J.J.; Descamps, M.; Collins, G., Molecular Mobility and Fragility in Indomethacin: A Thermally Stimulated Depolarization Current Study, Pharm. Res. 2001, 18, 1767.

${ }^{76}$ Bhugra, C. ; Shmeis, R.; Krill, S.L.; Pikal, M. J.; Different Measures of Molecular Mobility: Comparison between Calorimetric and Thermally Stimulated Current Relaxation Times 
Below Tg and Correlation with Dielectric Relaxation Times Above Tg, Pharm. Res. 2006, 23, 2277.

${ }^{77}$ Vyazovkin, S.; Dranca, I., Probing Beta Relaxation in Pharmaceutically Relevant Glasses by Using DSC, Pharm. Res., 2006, 23, 422-428.

${ }^{78}$ Götze, W. ; Recent Tests of the Mode-Coupling Theory for Glassy Dynamics, J. Phys. Condens. Matter 1999, 11, A1, 118.

${ }^{79}$ Johari, G. P.; Goldstein, M.; Viscous Liquids and the Glass Transition. II. Secondary Relaxations in Glasses of Rigid Molecules, J. Chem. Phys. 1970, 53, 2372.

${ }^{80}$ Bauer, Th.; Lunkenheimer, P.; Kastner, S.; Loidl, A.; Nonlinear Dielectric Response at the Excess Wing of Glass-Forming Liquids, Phys. Rev. Lett. 2013, 110, 107603.

${ }^{81}$ Samanta, S.; Richert, R.; Limitations of Heterogeneous Models of Liquid Dynamics: Very Slow Rate Exchange in the Excess Wing. J. Chem. Phys. 2014, 140, 054503.

${ }^{82}$ Samanta, S.; Richert, R., Nonlinear Dielectric Behavior of a Secondary Relaxation: Glassy D-Sorbitol, J. Phys. Chem. B, Article ASAP, doi: 10.1021/jp506854k.

${ }^{83}$ Wang, C. L.; Hirade, T.; Maurer, F. H. J.; Eldrup, M.; Pedersen, N. J.; Free-Volume Distribution and Positronium Formation in Amorphous Polymers: Temperature and PositronIrradiation-Time Dependence, J. Chem. Phys 1998, 108, 4654.

${ }^{84}$ Pazmino Betancourt, B.A.; Hanakata, P.Z.; Starr, F.W.; Douglas, J.F.; Quantitative Relations between Cooperative Motion, Emergent Elasticity, and Free Volume in Model Glass-Forming Polymer Materials, Proc. Natl. Acad. Sci. USA 2015, 112, 2966.

${ }^{85}$ Larini, L.; Ottochian, A.; De Michele, C.; Leporini, D.; Universal Scaling between Structural Relaxation and Vibrational Dynamics in Glass-Forming Liquids and Polymers, Nature Physics 2008, 4, 42.

${ }^{86}$ Puosi, F. Michele, C.D.; Leporini, D.; Scaling between Relaxation, Transport and Caged Dynamics in a Binary Mixture on a Per-Component Basis, J.Chem.Phys. 2013, 138,12A532.

${ }^{87}$ Buchenau, U.; Zorn, R.; Ramos, M. A.; Probing Cooperative Liquid Dynamics with the Mean Square Displacement, Phys.Rev.E 2014, 90, 042312.

${ }^{88}$ Fujimori, H.; Oguni, M. Correlation Index $\left(T_{\mathrm{ga}}-T_{\mathrm{gb}}\right) / T_{\mathrm{ga}}$ and Activation Energy Ratio as Parameters Characterizing the Structure of Liquid and Glass. Solid State Commun. 1995, 94, $157-162$.

${ }^{89}$ Bershtein V. A. ; Egorov, V. M.; Differential Scanning Calorimetry of Polymers, 1994, Ellis Horwood, New York. 
${ }^{90}$ Bershtein, V. A. ; Egorov, V. M. ; Emelyanov, Y.A.; Stepanov, V.A.; The Nature of $\beta$ Relaxation in Polymers, Polym. Bulletin, 1983, 9, 98-105.

${ }^{91}$ Bershtein, V. A.; Egorova, L. M.; Prud'homme, R. E. ; Peculiarities of the segmental dynamics in amorphous miscible polymer blends as a consequence of the common nature of $\alpha$ and $\beta$ relaxations, J. Macromol. Sci., Phys. 1997, B36, 513.

${ }^{92}$ Hristov, H. A.; Bolan, B.; Yee, A. F.; Xie, L.; Gidley, D. W.; Measurement of Hole Volume in Amorphous Polymers Using Positron Spectroscopy, Macromolecules 1996, 29, 8507-8516.

${ }^{93}$ Aji, D. P. B.; Johari, G. P; Kinetic-freezing and unfreezing of local-region fluctuations in a glass structure observed by heat capacity hysteresis, J. Chem. Phys. 2015, 142,214501.

${ }^{94}$ Duvvuri K. , Richert, R.; Dynamics of glass-forming liquids. VI. Dielectric relaxation study of neat decahydro-naphthalene, J. Chem. Phys. 2002, 117, 4414. 


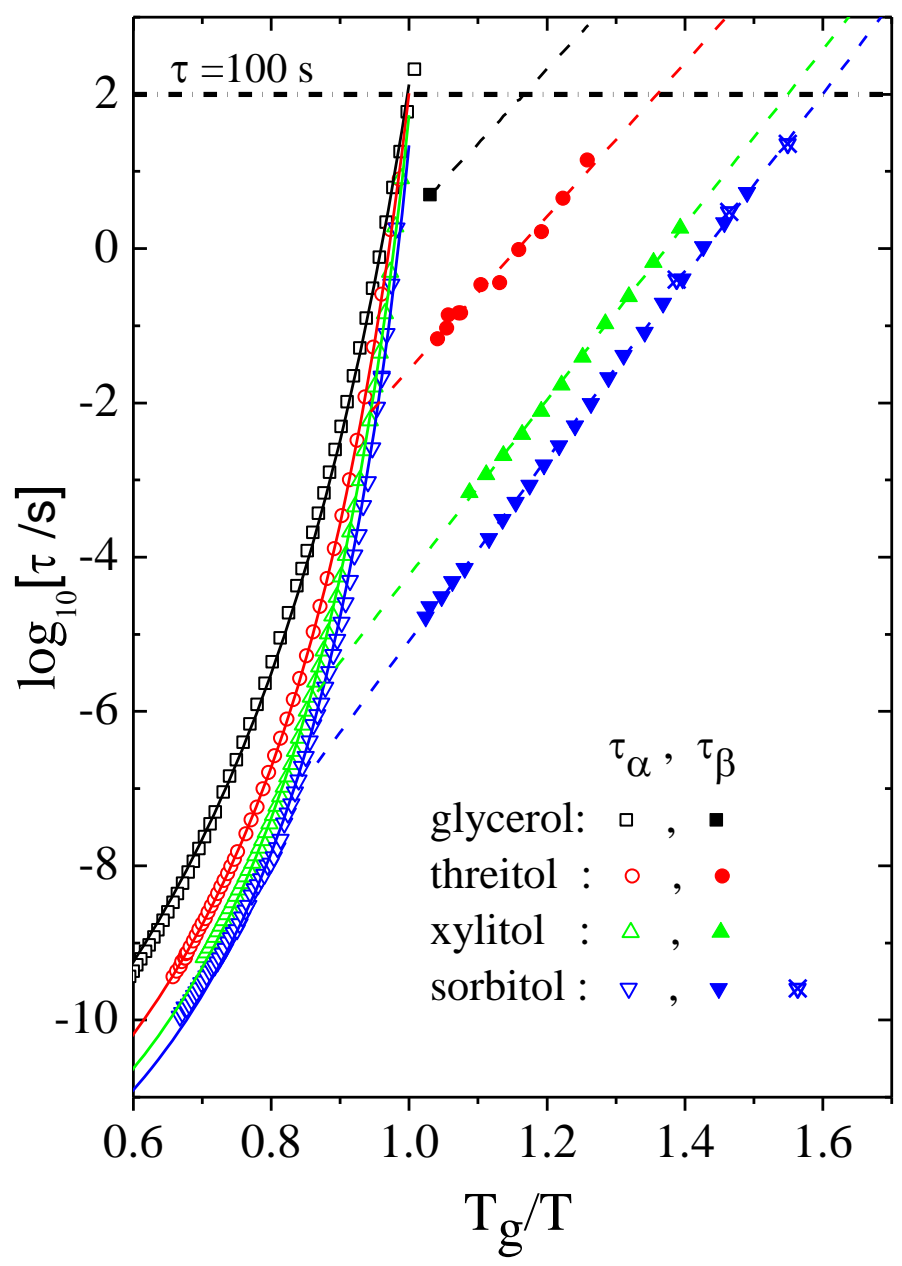

Figure.1: Relaxation map of logarithm of the $\alpha$ - (open symbols) and JG $\beta$-relaxation (filled symbols) times, $\tau_{\alpha}$ and $\tau_{\beta}$, against $T_{g} / T$ of the four polyalcohols adapted from the data of Do $\beta$ et al. ${ }^{69,70}$ and additional data from this study (crossed symbols). Squares, circles, up and down triangles are symbols for glycerol, threitol, xylitol and sorbitol, respectively. Continuous lines are from Vogel-Tammann-Fulcher fits to $\tau_{\alpha}$, dashed lines for the Arrhenius fit to $\tau_{\beta}$. The horizontal dotted-dashed line marks the glass transition as $\tau=100 \mathrm{~s}$. 


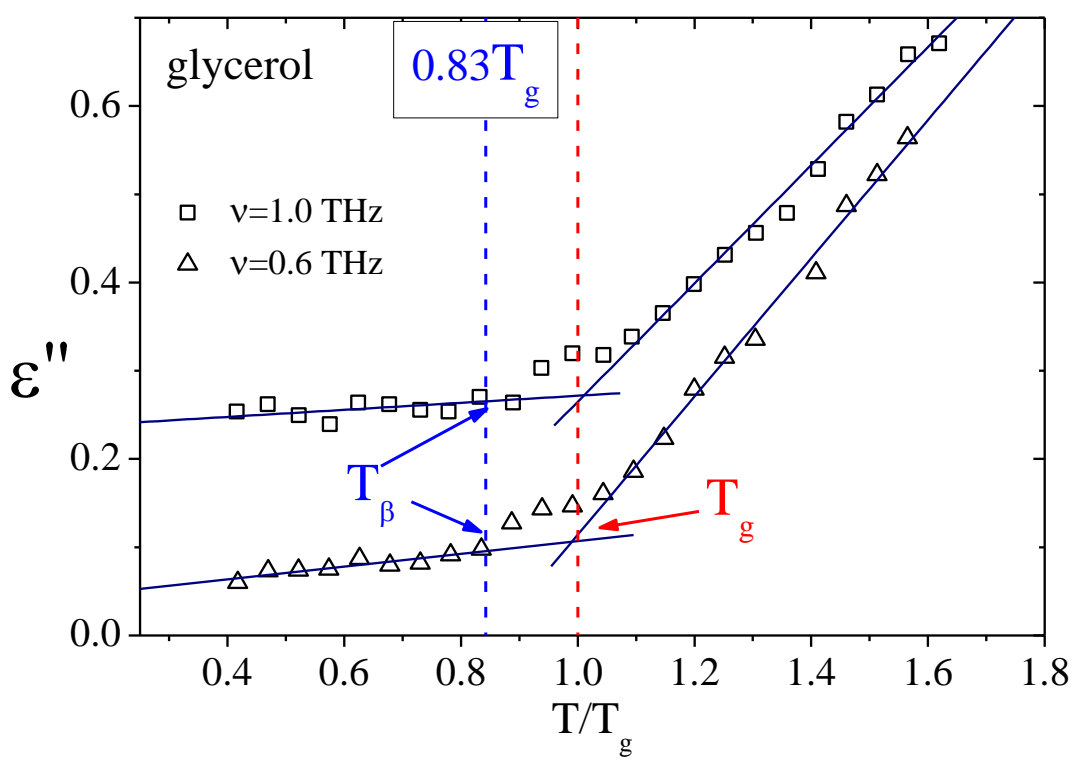

Figure 2: Dielectric losses of glycerol at $0.6 \mathrm{THz}$ (open triangles) and at $1 \mathrm{THz}$ (open squares) versus reduced temperature $T / T_{g}$. Data are from Ref.[60]. Straight lines are linear regressions to the low and high temperature data. Blue and red dashed vertical lines mark the occurrence of $\mathrm{T}_{\beta}$ and $\mathrm{T}_{\mathrm{g}}$ as shown in Figure 1. 


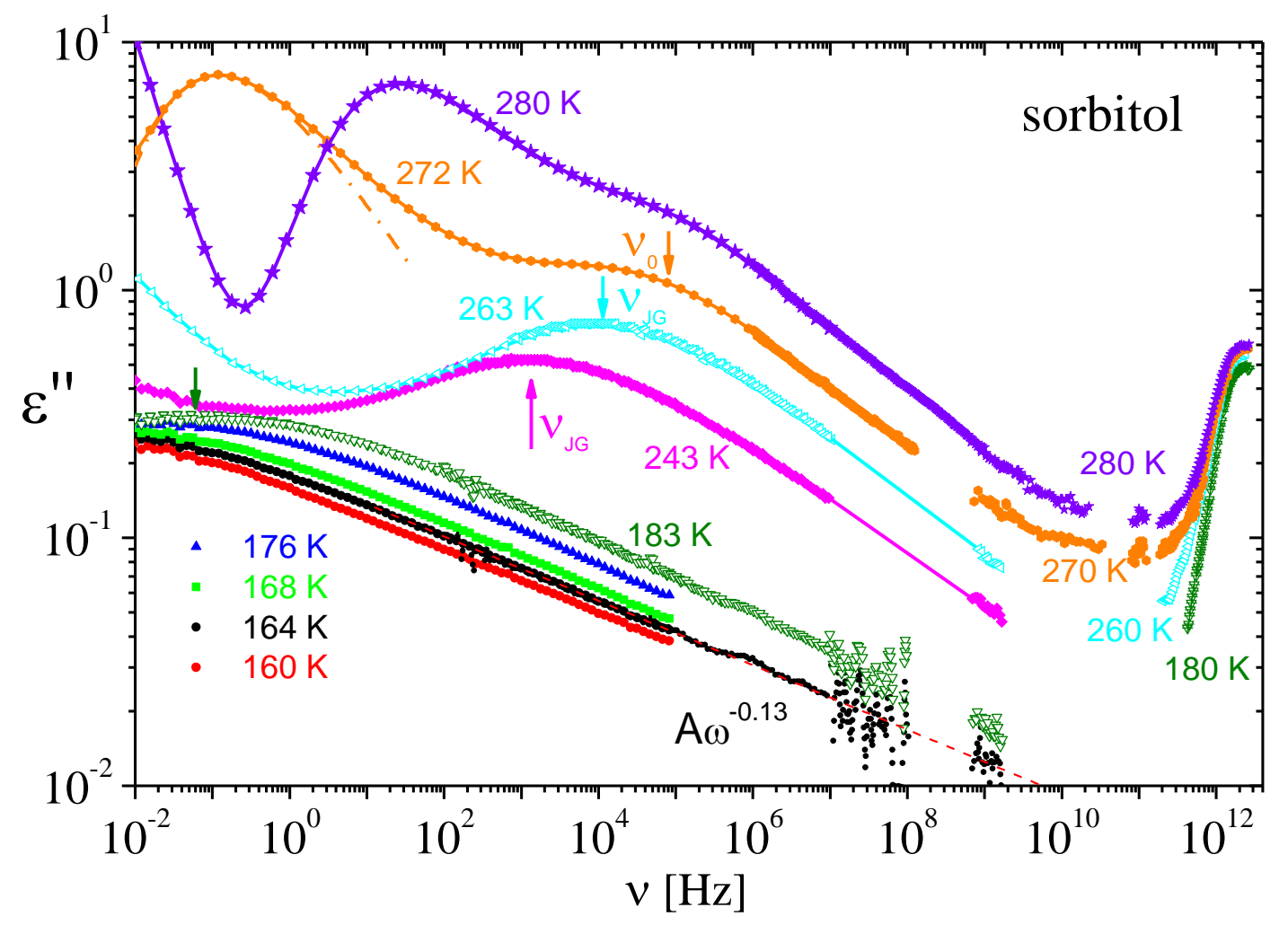

Figure 3: Dielectric loss spectra of sorbitol at selected temperatures ranging from below $T_{\beta}$ to above $T_{g}$. Dashed line is a power law fit of the high frequency flank of data at $164 \mathrm{~K}$. Dash-dotted line is a fit with a Fourier-transformed derivative of the Kohlrausch correlation function $(n=0.52)$. Vertical arrows mark the primitive relaxation frequency $v_{0}$ or the JG relaxation frequency $v_{\mathrm{JG}}$. Data are collected from this study $(v=10 \mathrm{mHz}-1 \mathrm{GHz}, \mathrm{T}=272,280 \mathrm{~K}$ and $v=10 \mathrm{mHz}-100 \mathrm{kHz}, \mathrm{T}=160,164,168,176,183,243,263 \mathrm{~K})$ and from ref.[63] ( $v=100$ Hz-3 GHz, T=164, 183, 243, $263 \mathrm{~K})$, ref.[23] ( $v=0.8 \mathrm{GHz}-1 \mathrm{THz}, \mathrm{T}=270,280 \mathrm{~K})$ and ref.[60] $(v=0.2-4 \mathrm{THz}, \mathrm{T}=180,260,270,280 \mathrm{~K})$. 

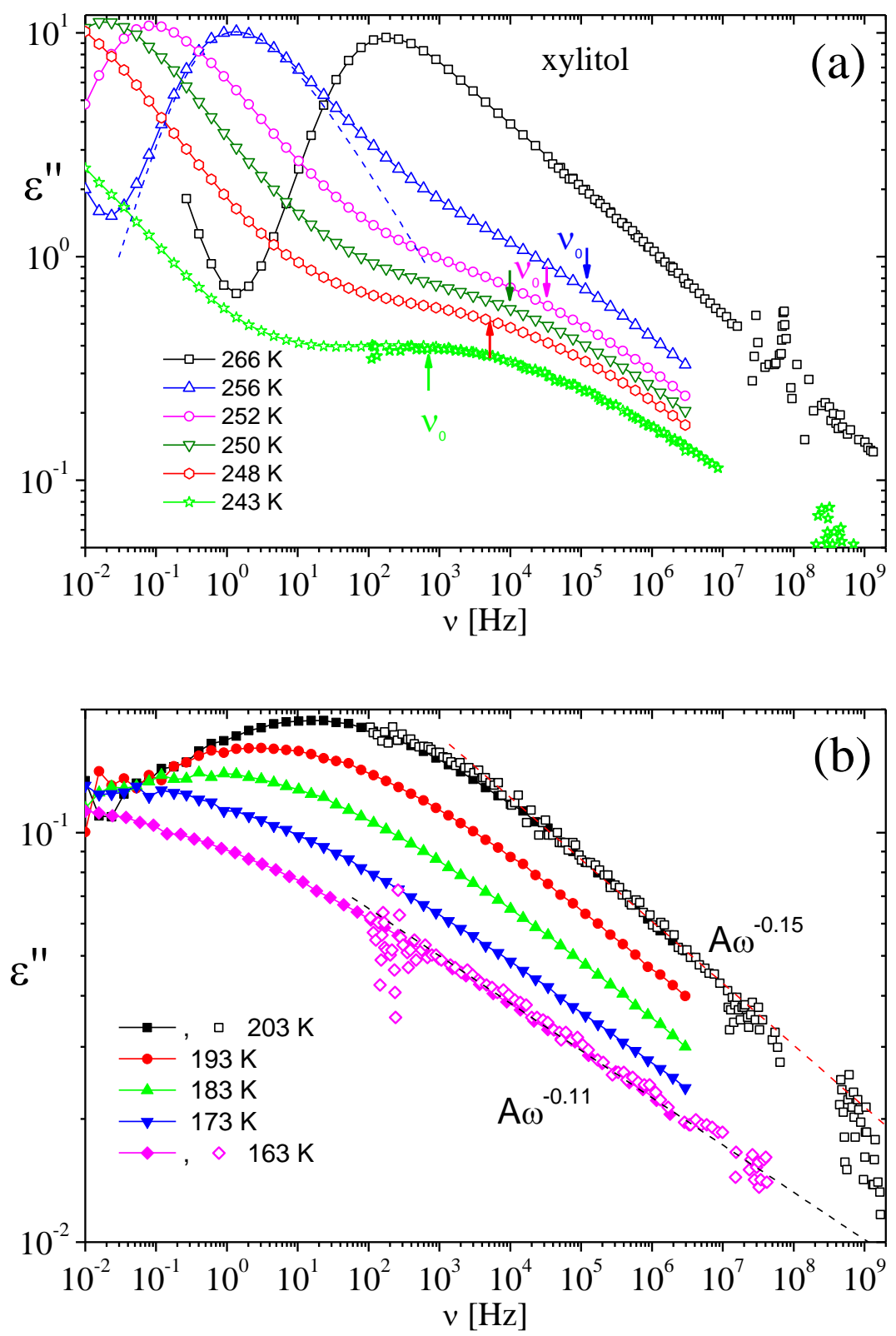

Figure 4: Dielectric loss spectra of xylitol at selected temperatures. Panel (a): from right to left: $266 \mathrm{~K}, 256 \mathrm{~K}, 252 \mathrm{~K}, 250 \mathrm{~K}, 248 \mathrm{~K}, 243 \mathrm{~K}$. The dashed line is a fit to the $\alpha$-relaxation peak by the one-sided Fourier transform of the Kohlrausch function with $n=0.46$. Each vertical arrow pointing toward certain data taken at some temperature indicates the location of the primitive relaxation frequency, $v_{0}$. Panel (b): from right to left: $203 \mathrm{~K}, 193 \mathrm{~K}, 183 \mathrm{~K}, 173$ $\mathrm{K}, 163 \mathrm{~K}$. The dashed lines are power law fits of the high frequency flank of data at $203 \mathrm{~K}$ and $163 \mathrm{~K}$. Data are collected from this study and from ref. [63] ( $v=100 \mathrm{~Hz}-3 \mathrm{GHz}, \mathrm{T}=163$, $203 \mathrm{~K})$. 


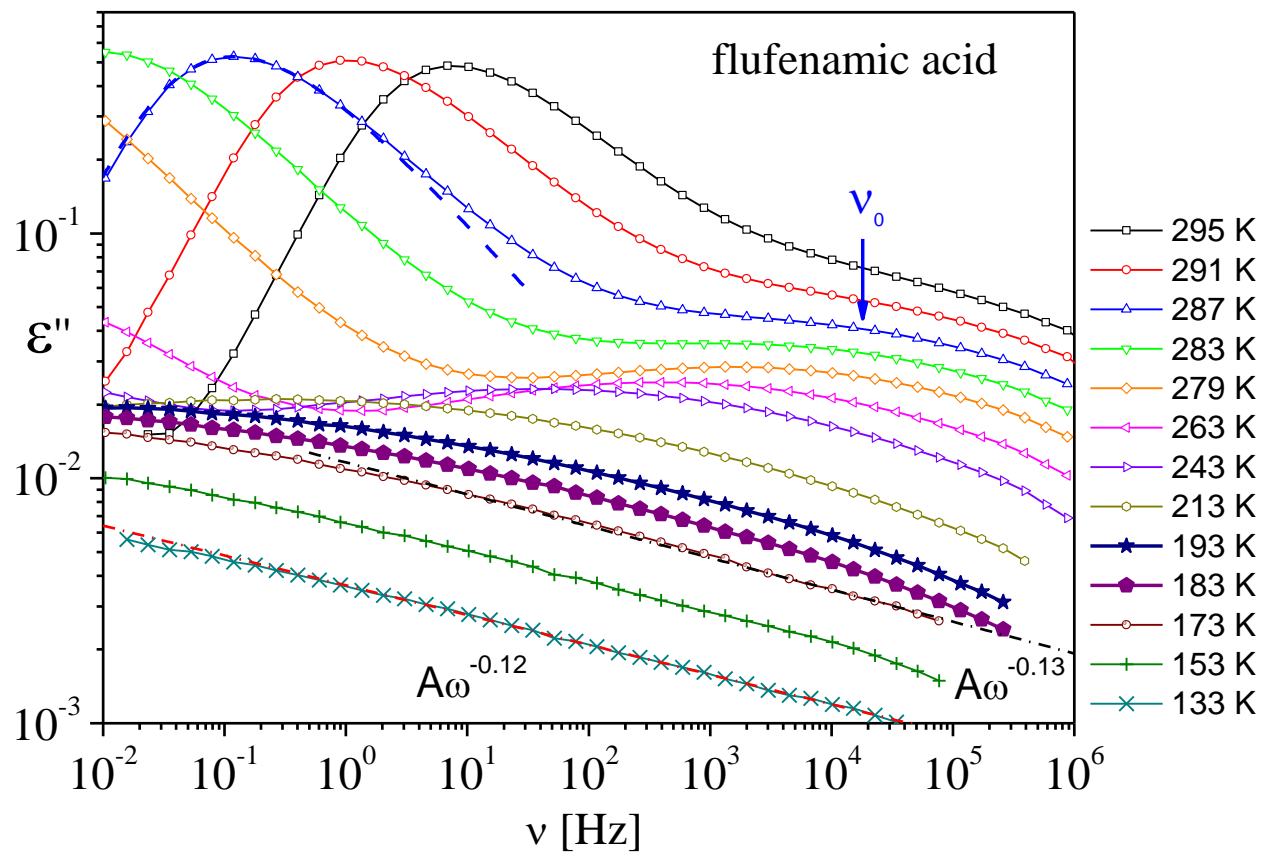

Figure 5: Dielectric loss spectra of flufenamic acid at selected temperatures. The dashed line is a fit to the $\alpha$-relaxation peak at $287 \mathrm{~K}$ by the one-sided Fourier transform of the Kohlrausch function with $n=0.46$. The vertical arrow mark the location of the primitive relaxation frequency, $v_{0}$. The dash-dotted lines are power law fits of the high frequency flank of data at $153 \mathrm{~K}$ and $173 \mathrm{~K}$. The two thick lines and bigger symbols are for $193 \mathrm{~K}$ and $183 \mathrm{~K}$ bracketing the $\mathrm{T}_{\beta}=189 \mathrm{~K}$. 


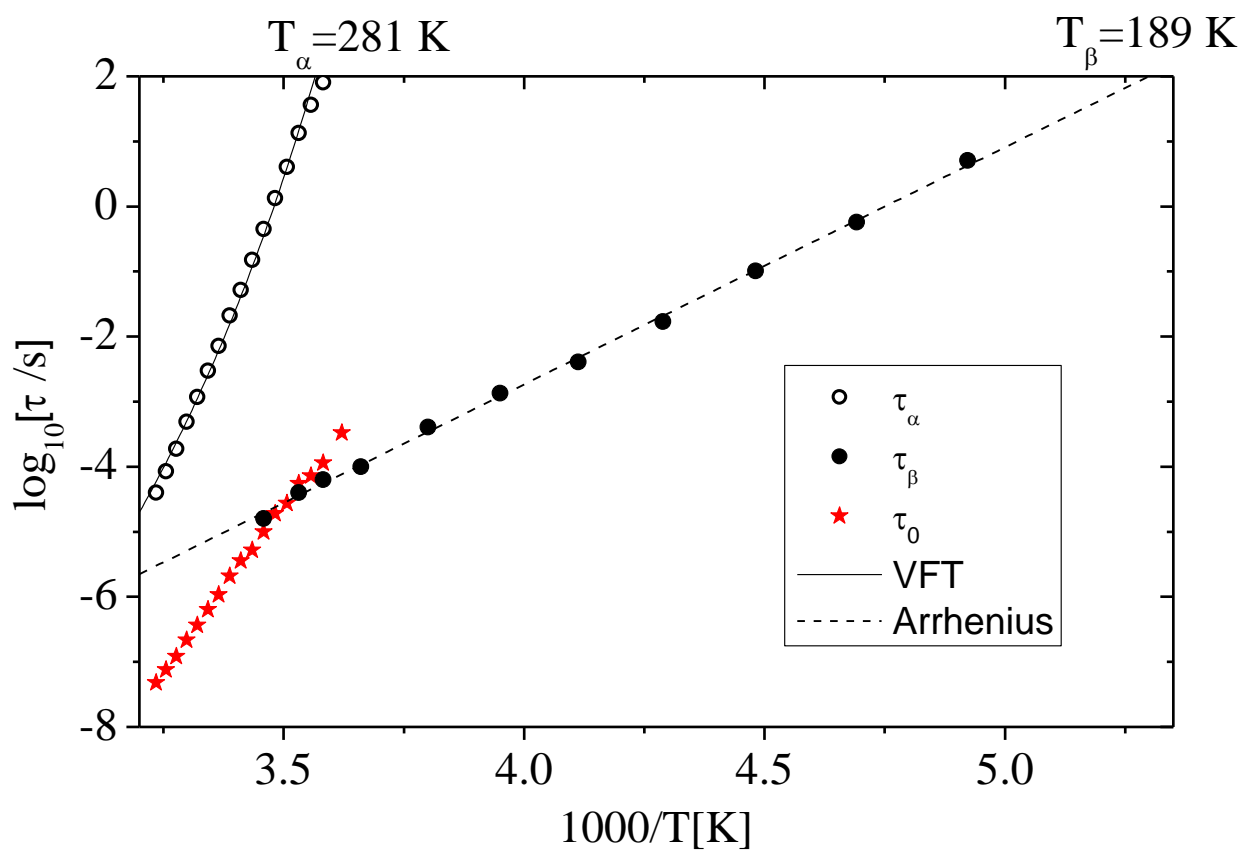

Figure 6:Relaxation map for flufenamic acid: $\alpha$ - (open circles) and JG $\beta$-relaxation (filled circles) times, $\tau_{\alpha}$ and $\tau_{\beta}$, plotted against reciprocal temperature. Red star symbols are for primitive relaxation times, calculated according to eq.(2). Continuous line is a VogelTammann-Fulcher fit to $\tau_{\alpha}$, dashed line is the Arrhenius fit to $\tau_{\beta}=5 \times 10^{-18} \exp (8400 / T) \mathrm{s}$. 